\title{
BANDWIDTH ALLOCATION ALGORITHM WITH USER MOBILITY DYNAMICS IN FEMTOCELL NETWORK
}

\author{
Nicholas O. Oyie, ${ }^{*}$ Philip K. Langat, ${ }^{*}$ and Stephen Musyoki**
}

\begin{abstract}
Fourth generation cellular networks provide ubiquitous broadband access to a growing number of mobile users worldwide. In this context, resource allocation in orthogonal frequency division multiple access femtocell network systems presents an important milestone towards realization of high-speed services. In this paper, we focus on impact of mobility dynamics on time/frequency allocation in femtocell network. We considered users' mobility dynamics taking into account the variation of their positions with time. We propose a scalable mobility-aware femtocell cluster-based resource allocation (M-FCRA) scheme based on cluster formation, clusterhead resource allocation with user mobility awareness, and resource contention resolution. M-FCRA formulates the frequency/time resources allocation mathematically as a min-max optimization problem and generates synchronized resource reservation requests that maximize bandwidth utilization and quality of service. The results show that M-FCRA performs better than femtocell clusterbased resource allocation (FCRA) algorithm at various signal to interference and noise ratio levels in terms of throughput satisfaction rate and spectrum spatial reuse.
\end{abstract}

\section{Key Words}

Clustering, femtocell, OFDMA, resource allocation, user mobility

\section{Introduction}

Mobile applications under fourth generation (4G) technology have been the driver behind customers' demand for more and more data. Extraction of high-quality services has been a challenge in recent years. One solution to enhance indoor coverage is the so-called femtocell access points (FAPs) or home base stations [1]. These are lowpower base stations designed for indoor usage that allow cellular network providers to extend indoor coverage where

* Department of Telecommunication and Information Engineering, Jomo Kenyatta University of Agriculture and Technology, Nairobi, Kenya; e-mail: oyie3nick@yahoo.com, kibetlp@ jkuat.ac.ke

** Department of Electrical and Electronic Engineering, Technical University of Kenya, Nairobi, Kenya; e-mail: smusyoki@yahoo.com

Recommended by Dr. Leone C. Monticone

(DOI: 10.2316/Journal.214.2014.3.214-1075) it is limited or unavailable. On the air interface, FAPs provide radio coverage to a given cellular standard such as global system for mobile communications, universal mobile telecommunications system, worldwide interoperability for microwave access, and long-term evolution (LTE), while the back-haul connection makes use of a broadband connection such as optical fibre or digital subscriber line. The use of femtocells benefits both users and operators by delivery of high quality of service (QoS).

In this paper, we present a scalable resource allocation algorithm called mobility-aware femtocell cluster-based resource allocation (M-FCRA) for orthogonal frequency division multiple access (OFDMA)-based femtocells. The use of OFDMA technology is motivated by the fact that next generation networks such as $4 \mathrm{G}$ apply it. The goal of this paper is to allocate the best set of time/frequency resources to each FAP so as to deliver the users data, while considering mobility of users and minimizing the difference between the required and allocated set of resources. A resource allocation is formulated mathematically as a MinMax optimization problem to achieve this. It is a hybrid of centralized/distributed algorithm called M-FCRA involving three stages: First, cluster formation, then cluster-head $(\mathrm{CH})$ time/frequency resources allocation with user mobility awareness, and lastly resource contention resolution among FAPs. First, M-FCRA uses a distributed approach to build disjoint clusters of femtocells. Then, a $\mathrm{CH}$ is elected within each cluster. $\mathrm{CH}$ assigns resources to all FAPs in its cluster taking into account their required bandwidth and mobility dynamics such as position with time [2].

Comparison of M-FCRA with an existing solution, FCRA, to evaluate its efficiency is performed [2]. Evaluation and comparison metrics are the throughput satisfaction rate (TSR) and spectrum spatial reuse (SSR). The simulation results obtained show that M-FCRA algorithm that incorporates user mobility dynamics outperforms the FCRA algorithm for low signal to interference plus noise ratio (SINR) as the latter does not consider user mobility for both small- and large-sized networks.

\section{Related Work}

The indoor access in LTE networks is supported by the deployment of femtocells. Because femtocells are designed to 
be deployed by user demand, resource allocation problems are severe. In terms of resource management, [3] a framework to allocate different resources with different users' requirements is suggested. In [4] a dynamic interference avoidance scheme to coordinate a group of neighbouring cells is presented. However, this scheme was designed for macrocell base stations. In femtocell networks, the design of cognitive femto network was studied.

In [5] a cognitive base station that can exploit their knowledge of the radio scene for interference management is suggested. Implementation of cognitive femtocell base stations for resource allocation by using a game-theoretic framework is proposed in [6]. With cognitive femtocell base stations, [7] insertion of sensing frames to scan the whole wireless resources periodically is recommended. However, when sensing the whole wireless resources, the femtocell cannot receive and transmit data simultaneously. Moreover, cognitive base station is required, and cognitive radio capabilities need to be incorporated into the femtocell base stations. In addition [8], a presentation of a graph colouring-based dynamic sub-band allocation (GC-DSA) as a GC-DSA technique to avoid downlink interference is made. In [9], a graph-based dynamic frequency reuse as a resource allocation method based on graph colouring is developed. With graph colouring algorithms, the assignment of physical resource blocks (PRBs) to a femtocell is restricted as a vertex can only be assigned a single colour.

The dynamic frequency planning (DFP) is also proposed in [3] to decrease interference and reuse available sub-channels in OFDMA networks. An adaptive fractional frequency reuse (FFR) algorithm is proposed in [10], where the femtocell management system (FMS) plans the coverage areas of femtocells according to the minimum acceptable signal strength of femtocells. The FMS allocates usable resources for every femtocell in accordance with the number of cliques, which is formed by the union of neighbouring femtocells. In [11], a proposal that each femtocell adopted a modulo-prime function and an interference topology to randomly access some frequency bands is presented. The proposed distributed random access scheme is specifically designed for co-channel and co-tiered deployment. However, different users might be allocated to the same wireless frequency bands with modulo-prime, where different users will acquire the interfering resources. The approaches presented in [3], [11], [12] allocate fixed sub-channels to a femtocell. A sub-channel is composed of a number of PRBs within the same frequency band. Depending on traffic requirements, certain PRBs in the allocated sub-channels may be used by the femtocell. In case of underutilization of PRBs in the sub-channels, PRBs are wasted as they cannot be reused by other femtocells [10].

A decentralized frequency-ALOHA (F-ALOHA) spectrum allocation strategy for two-tier cellular networks is described in [13]. The strategy is based on spectrum split between the macrocell and femtocells. As it is based on a pseudo-random algorithm, F-ALOHA cannot guarantee any degree of QoS. Furthermore, this approach does not consider time-frequency slots as resources. However, it focuses on sub-carriers allocation.
Three resource allocation algorithms in OFDMA femtocells are proposed in [14]. The first method is called orthogonal assignment algorithm. It splits the bandwidth into two independent sets $B_{M}$ and $B_{F}$ which are used by the macrocells and femtocells, respectively. The challenge is to associate the best split that maximizes the satisfaction of the required QoS to end users. However, femto-tofemto interference is not taken into account in this scheme. Moreover, it remains an important factor for indoor performance, especially when femtocells are highly populated.

In [15], the authors investigated the radio resource utilization efficiency in wireless mesh networks. They proposed two clustering schemes to improve the resource utilization in such networks.

The main focus in clustering in ad hoc networks is efficient handling of the frequent network topological changes. This is as a result of ad hoc nodes mobility. The objective has been to conform quickly to changes in topology, which hardly happens in femtocell networks, because of their relatively static topologies [2].

Radio resource allocation in OFDMA-based femtocell networks is an ongoing research area. In [2], FCRA based on clustering is proposed. It is a scalable resource allocation strategy. As described in [2] a distributed clustering algorithm to form disjoint femtocell clusters is presented. The resource allocation problem is sub-divided into sub-problems by clustering. It includes also the use of optimum centralized spectrum allocation in each cluster to allocate more efficiently the available resources. However, FCRA does not consider user mobility. It was assumed that all users were static.

In this paper, we present a scalable approach with user mobility prediction/awareness. The resource allocation problem in OFDMA femtocell network has been formulated mathematically. The mathematical problem is subsequently solved by mixed integer linear programming method using IBM ILOG CPLEX solver.

\section{Problem Description}

This paper considers a macrocell embedded with a set $F$ of femtocells (FAPs) network as shown in Fig. 1. FAPs and macrocells are assumed to use OFDMA technology for operation. We consider an OFDMA frame structure that is made up of time-frequency slots, known as tiles [11]. In this work, we focus on the downlink communications. An assumption is made that operating bandwidth is split between the macrocell and femtocells, hence there is limited interference between femtocell and macrocell users in the network [11], [16].

The LTE system is composed mainly of two parts: the air interface known as the evolved-universal terrestrial radio access network (E-UTRAN) and the packet switched core network called evolved packet core. The evolved NodeB (eNB) is the only node of the E-UTRAN from the network side.

It is responsible for the provision of network connectivity through the air interface to all user equipments (UEs) in the cell, according to the classic cellular network paradigm [17]. 
The radio interface supports both frequency and time division duplexing at the physical layer. Channel access, instead, is based on OFDMA. OFDMA provides high flexibility in terms of scheduling and interference management [18].

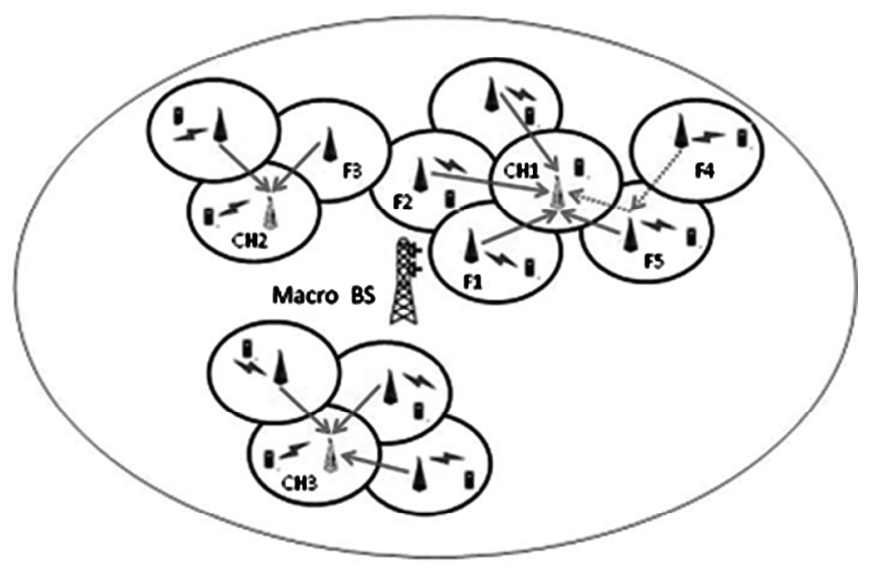

Figure 1. Network model.

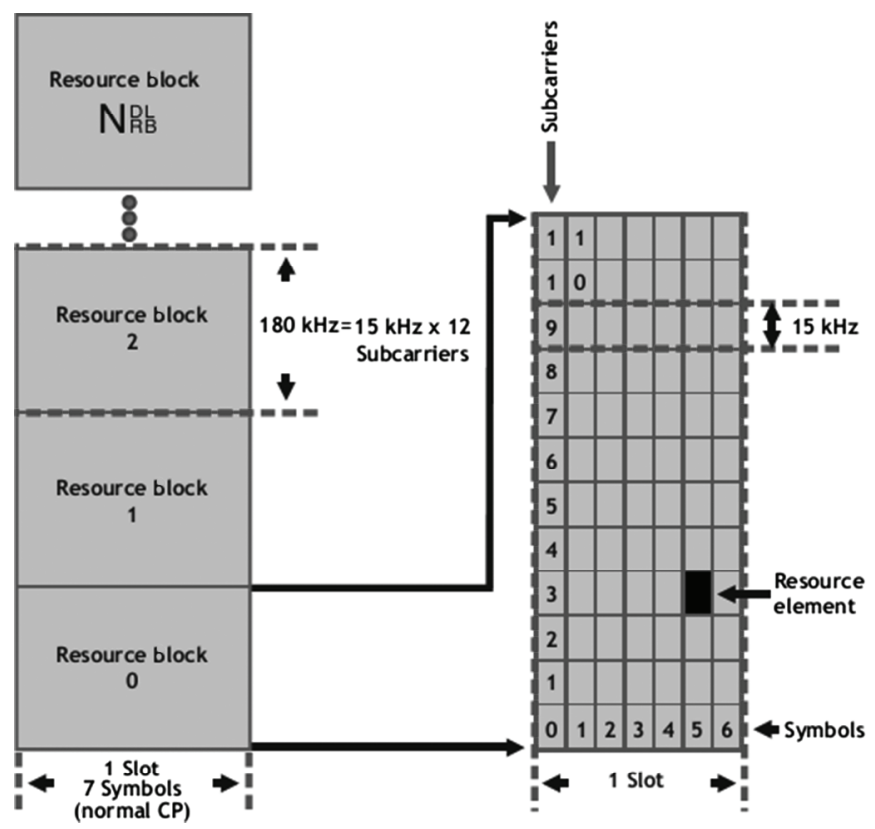

Figure 2. OFDM RB structure.

According to the third generation partnership project [19], bandwidth is allocated in time/frequency domain. Radio resources are distributed every transmission time interval (TTI), each one lasting $1 \mathrm{~ms}$ in time domain. Each TTI is composed of two time slots of $0.5 \mathrm{~ms}$. It corresponds to seven OFDM symbols in the configuration with short cyclic prefix (i.e. one OFDM symbol corresponds to $71.3 \mu$ s including normal cyclic prefix); 10 consecutive TTIs form the LTE Frame lasting $10 \mathrm{~ms}$. Radio resource is divided into $180 \mathrm{kHz}$ sub-channels in the frequency domain, corresponding to 12 consecutive and equally spaced subcarriers (i.e. $15 \mathrm{kHz}$ spacing per sub-carrier) as shown in Fig. 2. A time/frequency resource time slot lasting $0.5 \mathrm{~ms}$ in the time domain and over one sub-channel in the frequency domain is called a resource block (RB) or tile as commonly used in this paper. RB or tile corresponds to the smallest radio resource that can be allocated for data transmission. Due to the fixed sub-channel size, variation of the number of sub-channels depends on the system bandwidth configurations, for example 25 and $50 \mathrm{RBs}$ for system bandwidths of 5 and $10 \mathrm{MHz}$, respectively, [17].

For each femtocell $F_{a} \in F$, the set of defined interfering femtocells is denoted by $I_{a}$. This set depends on the indoor path loss model and minimum required SINR values in the network [2]. As in [14], the minimum required SINR is modelled based on the A1-type generalized path loss models for the frequency range $2-6 \mathrm{GHz}$ developed in WINNER II [20]. The min-max optimization problem for M-FCRA can be formulated as illustrated in Problem 1.

In addition, this paper defines for each femtocell $F_{a}$ the binary resource allocation matrix denoted by $\Delta_{a}$, with 1 or 0 in position $(i, j)$ according to whether the tile $(i, j)$ is used or not.

Problem 1 Min-Max femtocells resource allocation problem

$\begin{array}{ll}\forall F_{a} \in F: & \min \left[\max _{a}\left(\frac{R_{a}-\sum_{i, j} \Delta_{a}(i, j)+\left(R_{b}\right) p}{|F| \times R_{a}}\right)\right] \\ \text { Subject to: } & \sum_{i, j} \Delta_{a}(i, j) \leq R_{a} \\ \forall F_{a} \in F: & \\ \forall i, j, & \Delta_{a}(i, j)+\Delta_{b}(i, j) \leq 1 \\ \forall F_{a} \in F, \forall F_{b} \in I_{a}: & \Delta_{a}(i, j) \in\{0,1\} \\ \forall_{i, j,} \forall F_{a} \in F: & \\ p \in\{0,1\}: & \end{array}$

To represent the users' demands, a vector $V_{a}$ is introduced. The vector elements correspond to the radio resources required by users connected to femtocell $F_{a}$. The total number of RBs required by the femtocell $F_{a}$ to fulfill the attached users' demands is denoted by $R_{a}$ such that $R_{a}=\sum_{i=1}^{n_{a}} V_{a}(i)$, where $n_{a}$ is the total number of users belonging to femtocell $F_{a}$. Obviously, $R_{a}$ is not constant and depends on the arrival/departure process of end users. Hence, assumption made was that $R_{a}$ is updated periodically every epoch $\delta_{t}[2]$.

End users associated with each femtocell follow a random distribution with a maximum value of 6 per femto. Each FAPs has two users attached to it at the beginning of simulation. Moreover, assumption was made that femtocells apply the round Robin approach to serve the connected users, [11], [14].

The main objective will be to minimize the maximum difference between the number of allocated and required resources in each FAP, given the set of interferer femtocells $I_{a}, \forall F_{a} \in F$ and for every epoch $\delta_{t}$. Condition (1) denotes that the resource scheduler must guarantee that femtocells cannot be allocated more than the required resources, (2) ensures that two interfering femtocells cannot use the same $\mathrm{RB}$, and condition (4) is the probability of connection of user $i$ to the next FAP in the network. 


\section{Proposed Scheme: M-FCRA}

The objective of M-FCRA is to incorporate user mobility awareness in the resource allocation problem. The femtocell network through the $\mathrm{CH}$ should be able to predict the next femtocell a user is likely to connect to. This probability of connection is the element of user mobility in the network. The probability is either 1 or 0 depending on the level of signal strength received by a user in the network. It also depends on the history of the user mobility pattern as transmitted to its serving femtocell.

Mobility prediction guarantees continuous services to mobile users, and efficient resource allocation in femtocell network. Mobile operators try to know the next mobile's location using mobility prediction. It consists of locating in advance the next connection point to anticipate resource allocation at the predicted point. The considered mobility prediction model is based on Markov chains, exploiting the mobility trace of the mobile user, in the context of LTE architecture in [21], [22]. It exploits a sequence of femtocells that a mobile user crosses during an interval of time. This model has been proved to be efficient enough to predict the movement changes of a mobile user. The model is based on two complementary prediction algorithms, the global prediction algorithm (GPA) and the local prediction algorithm (LPA). GPA allows predicting the regular movement of a user. It is based on the mobile client's mobility trace and a second-order continuous-time Markov chain, whose discrete states are the cells of the network. It computes the transition probabilities from its current cell to each cell in its neighbourhood. In case GPA fails, LPA uses a first-order continuous-time Markov chain. For complete mobility prediction, every cell entered, the algorithm computes the transition probability to every cell in the neighbourhood. If there is enough information in the mobility trace to predict the next cell, then GPA is used, otherwise LPA is used. The prediction procedure needs the knowledge of adjacent cells that is provided by the operator's network topology or by analysing the strength of received signals. The M-FCRA problem is formulated with connection probability $p \in\{0,1\}$ and $R_{b}$ is the requested resource in femtocell $F_{b}$ as it is shown in M-FCRA algorithm in Fig. 3 [23].

\subsection{Network Topology}

In the simulator, femtocell is represented by geometric properties and it is used for detailing the position and the ID of a femtocell. The building is composed of a number of apartments, each one delimiting the area of a given femtocell.

As defined in [15], a $5 \times 5$ apartment grid is considered as in Fig. 4. The building is composed of 25 apartments which are located over a $5 \times 5$ grid. A unique ID is used to identify each building, and its position is defined by a Cartesian system. Each squared form apartment has an area of $100 \mathrm{~m}^{2}$. Generally, each apartment contains at least one active FAP, that is, an active home eNB (HeNB) in each femtocell. For instance, a $5 \times 5$ grid building can contain up to 25 femtocells active HeNB. The probability

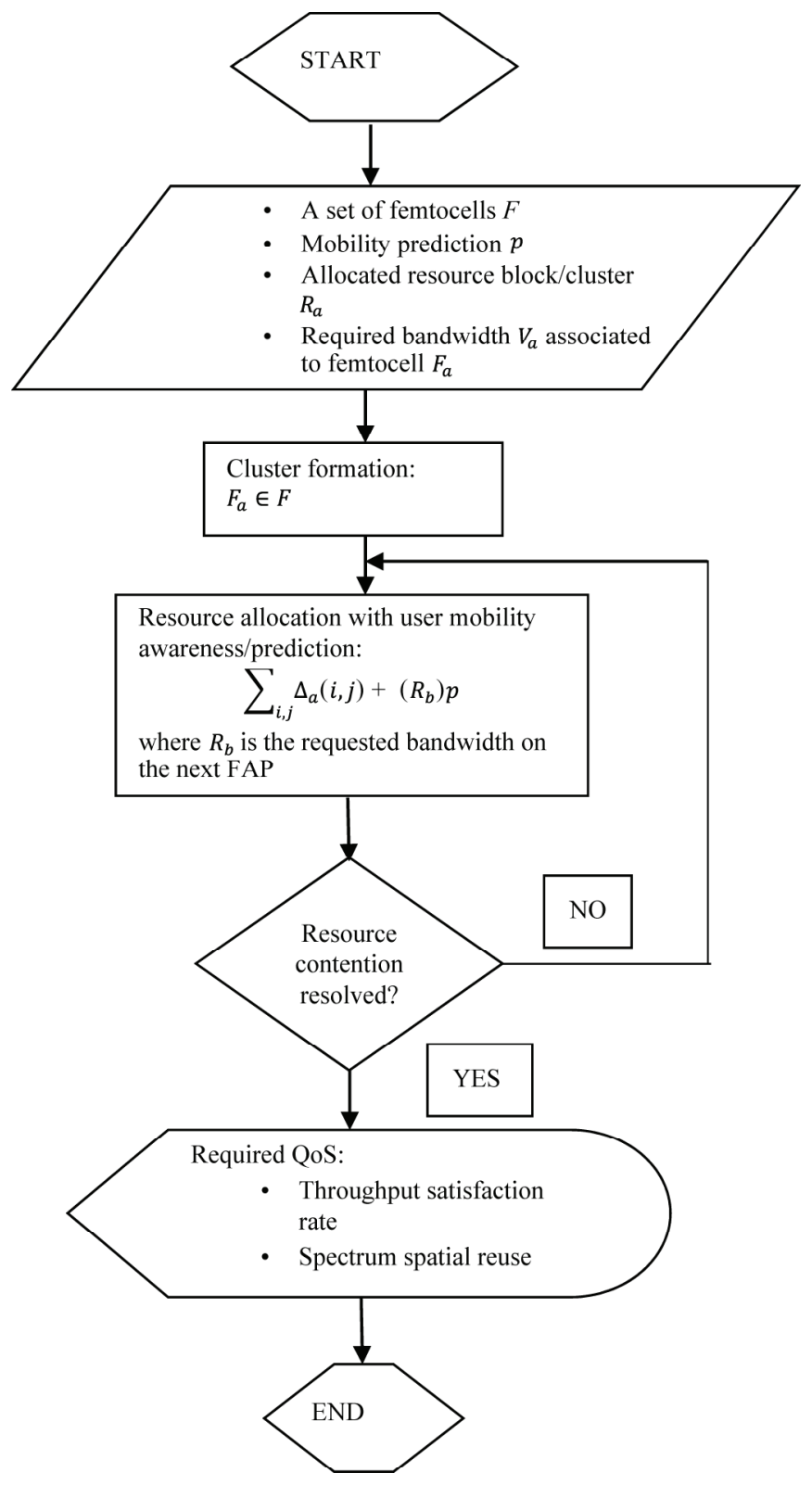

Figure 3. M-FCRA algorithm.

that an active home base station is present in an apartment can be randomly decided through the definition of an activity ratio [24]. Activity ratio is the ratio of active to inactive femtocells in the building at any given time.

\subsection{Single Macrocell with Femtocells Scenario}

This scenario models a single cell with a number of overlapping femtocells. The number of femtocells per building is equal to 25 and the total number of femtocells in the scenario is equal to $25 \times$ number of buildings. The parameters used are listed in Table 1.

The simulator logs all events to the standard output according to the debug level set to study a certain performance criteria. In addition, the log file provides the Cartesian position of all cells and UEs, the channels allocated to each eNB, as well as the unique ID that each UE and eNB is assigned [25]. A large log file is generated and the output is directed to a file and post-processing is done via 


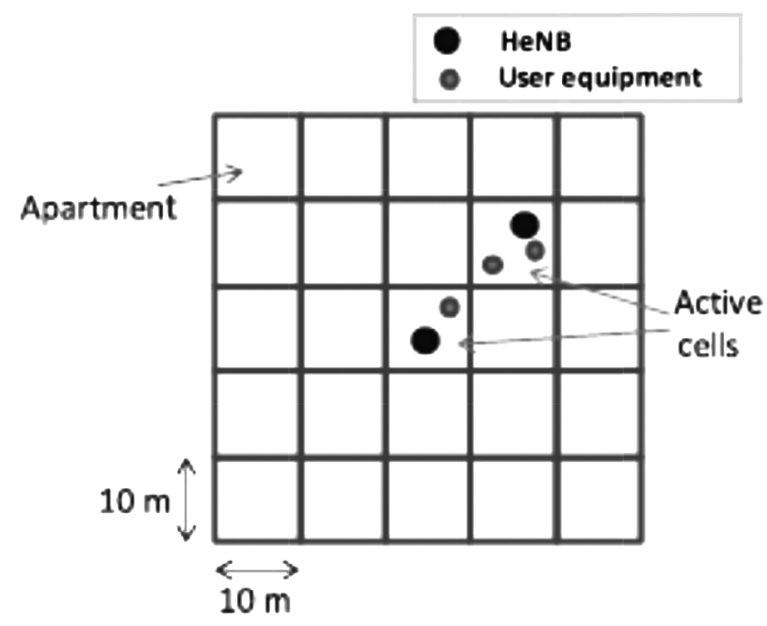

Figure $4.5 \times 5$ apartment grid.

Table 1

Parameters Used

\begin{tabular}{|l|l|}
\hline Parameter & Value \\
\hline Cell radius & $1 \mathrm{~km}$ \\
\hline Number of buildings & 1 \\
\hline Building type & $5 \times 5$ apartment grid \\
\hline Activity ratio & 1 \\
\hline Number of macrocell UE & 0 \\
\hline Number of femtocell UE & $2-6$ \\
\hline Traffic & VIDEO, VoIP, BE \\
\hline Scheduling type & Proportional fair \\
\hline Frame structure & FDD \\
\hline Bandwidth & $10 \mathrm{MHz}$ \\
\hline Speed & $0 \mathrm{~km} / \mathrm{h}$ for FCRA \\
& $3 \mathrm{~km} / \mathrm{h}$ for M-FCRA \\
\hline Access policy & Open \\
\hline MaxDelay & $0.1 \mathrm{~s}$ \\
\hline Video bit rate & $128 \mathrm{kbps}$ \\
\hline
\end{tabular}

FDD - frequency division duplex.

grep, sed, and awk to filter and print relevant performance metrics.

A sample of simulator output is: TX INF_BUF ID 104 B 14 SIZE 290 SRC 0 DST 5 T 1.709. This logs a transmission event for an infinite buffer source with packet ID 104 for bearer 14 . The packet size is 290 bytes and source is node 0 and destination is 5 . The event occurs at time 1.709. Similarly, a receive event is logged as follows: RX INF_BUF ID 106 B 14 SIZE 290 SRC -1 DST 5 D 0.001 .

\section{Performance Metrics}

We evaluate performance of M-FCRA based on the output of the optimization problem resolution for each constructed cluster. Two QoS metrics are considered:

(i) TSR

(ii) SSR

\subsection{Throughput Satisfaction Rate (TSR)}

TSR is the average level of satisfaction of a FAP with respect to the requested radio resources. For each femtocell $F_{a}, \operatorname{TSR}\left(F_{a}\right)$ is given by averaging the received number of allocated RBs to the total requested ones. It is expressed as follows:

$$
\operatorname{TSR}\left(F_{a}\right)=\left(\frac{\sum_{i, j} \Delta_{a}(i, j)}{R_{a}}\right)
$$

The TSR metric is thus given by:

$$
T S R=\sum_{F_{a} \in F}\left(\frac{T S R\left(F_{a}\right)}{|F|}\right)
$$

\subsection{Spectrum Spatial Reuse (SSR)}

SSR is the average number of femtocells using the same elementary RB within the femtocell network. Hence, it is defined as the mean value of RBs' (tiles) spatial reuse.

The $S S R$ metric is thus expressed as follows:

$$
S S R=\frac{1}{M \times|F|} \sum_{i, j} \sum_{F_{a} \in F} \Delta_{a}(i, j)
$$

where $M$ is equal to the length of OFDMA downlink frame expressed as tiles (time-frequency slots).

\section{Performance Analysis}

FCRA scheme was used as a benchmark to which the M-FCRA potential benefits were compared. The gain of M-FCRA was studied when the users were mobile in uniformly distributed FAPs and under various interference level scenarios. The mathematical problem solved by mixed integer linear programming method using the solver "ILOG CPLEX" [26]. It optimized the resource allocation under various constrains. Simulations were run and mean value calculated. The number of mobile equipment connected to each femtocell and their radio resource demands were varied in each simulation with time and position in the building for M-FCRA scheme while static for FCRA.

The analysis was achieved using an OFDMA downlink frame consisting of $M=50$ tiles (time-frequency slots), as in [3]. Users were distributed randomly within the femtocells with a maximum value of 6 per FAP [2]. Each user randomly generated its traffic demand (required bandwidth), which was translated into a certain number of tiles $V_{a}\left(0 \leq V_{a} \geq 8\right)$. Different SINR values were considered. The $F$ femtocells were distributed uniformly in a 


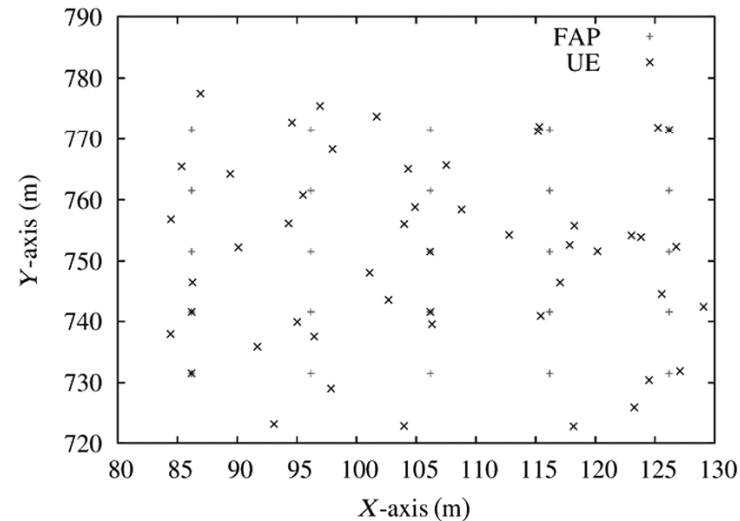

(a)

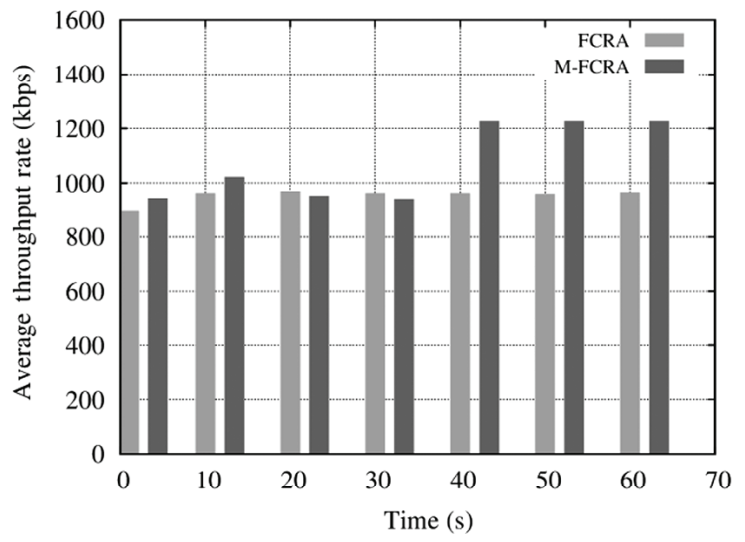

(c)

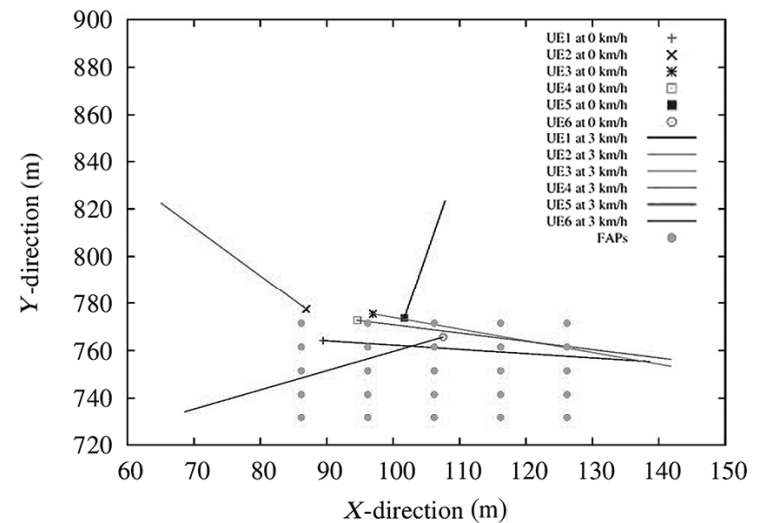

(b)

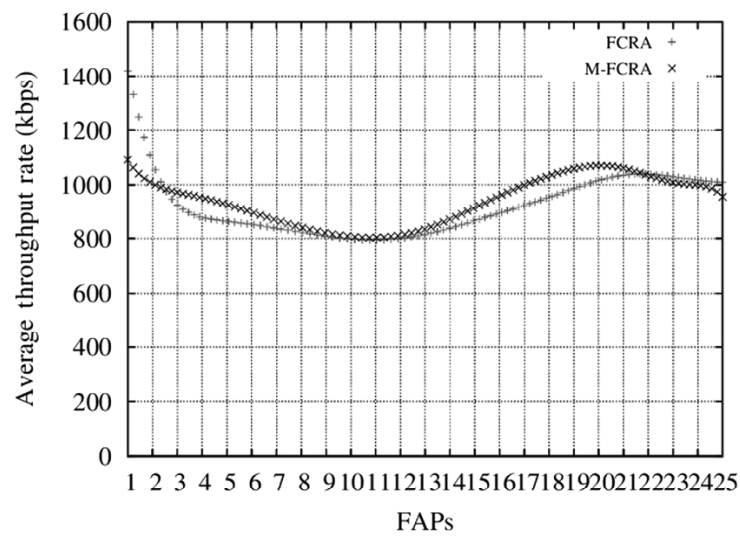

(d)

Figure 5. Simulation results: (a) Random distribution of users and uniform distribution of FAPs; (b) Trajectory of some users; (c) Average throughput rate as a function of time; and (d) Average throughput of each FAP.

2-D $50 \mathrm{M} \times 50 \mathrm{M}$ building, with one FAP uniformly placed in each $10 \mathrm{M} \times 10 \mathrm{M}$ room. Based on the SINR values and the path loss model [6], the interference matrix $I_{a}$ for every femtocell $F_{a}$ was derived. In the simulations, different SINR thresholds, $10,15,20$, and $25 \mathrm{~dB}$, were considered to indicate the degree of impact of the interference on the analysed metrics.

M-FCRA algorithm used a Bernoulli distribution to work out the resource contention resolution. In case of same tile assignment hence collision, each femtocell tries to resolve contention on the collided tiles by sampling a Bernoulli distribution. Subsequently, it rules whether the attacked user would continue using the tile or would transfer it from allocated resources. For simulations, the mean value of Bernoulli was set to 0.5. It follows that, femtocells have the same opportunity to use or discard the contented RB (tile). The results were concerned with the TSR and the SSR.

Figs. 5(a)-(d) illustrate simulation results of single cell with femtocells scenario. We considered a scenario with randomly and uniformly distributed UE and femtocells, respectively, as in Fig. 5(a).

Distance in metres along the $Y$ and $X$ axes indicates the position of the building with respect to the overlying macrocell. The femtocells are inside a building where macrocell coverage is poor. At the beginning of the simulation, each FAP has two users attached to it.

We considered the FCRA scheme where UEs are static, while the M-FCRA scheme with UEs moving at $3 \mathrm{~km} / \mathrm{h}$ in our simulations. Fig. 5(b) shows the approximate trajectory of each mobile user in the network after $60 \mathrm{~s}$. The direction of the users is random in the building. As illustrated, some users walk out of the building, while others into the building. We plotted the average TSR against time as shown in Fig. 5(c). It improves for M-FCRA and remains constant for FCRA. In the case of users moving closer to the FAPs they are connected, path loss reduces and hence SINR increases. This improves the SINR for the M-FCRA scheme, thus better throughput on higher SINR.

As users under FCRA are static, its SINR largely remains constant. Therefore, its average throughput with time remains almost the same. After $30 \mathrm{~s}$ of connections, M-FCRA outperforms FRCA by about $35 \%$. Consequently, users get better QoS on M-FCRA scheme.

Fig. 5(d) shows the average throughput taken per FAP within $60 \mathrm{~s}$. The graph indicates that FAPs at the centre of the building performed better in terms of average throughput rate on M-CRA than those at the edge, that is, $1,2,22,23,24$, and 25 . This was attributed to low 


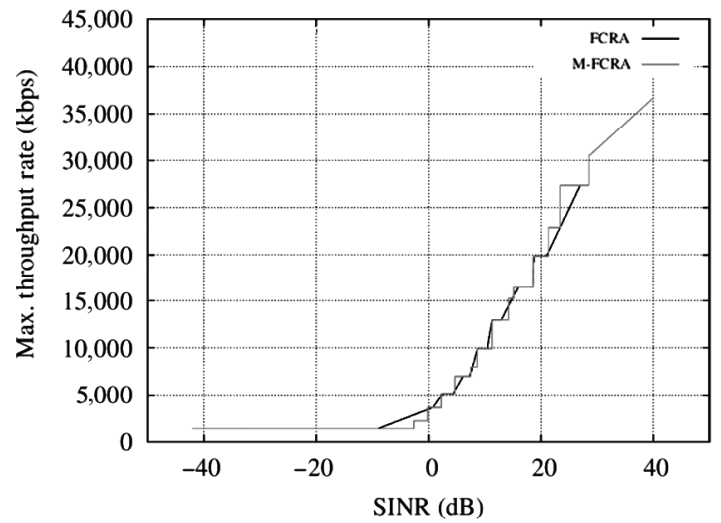

(a)

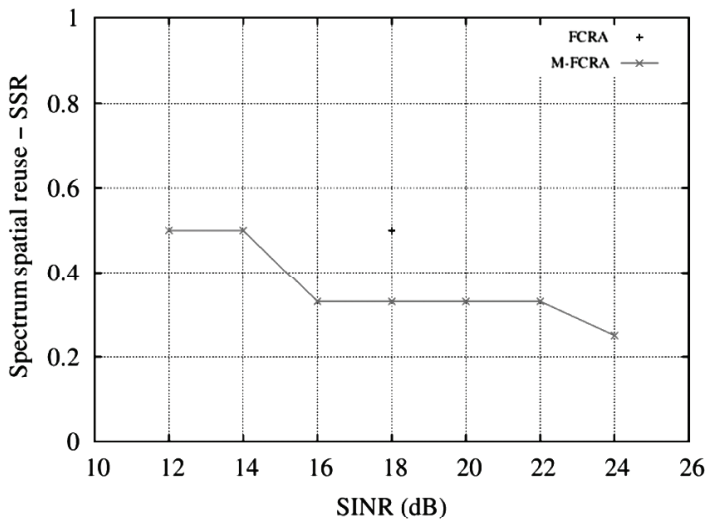

(b)

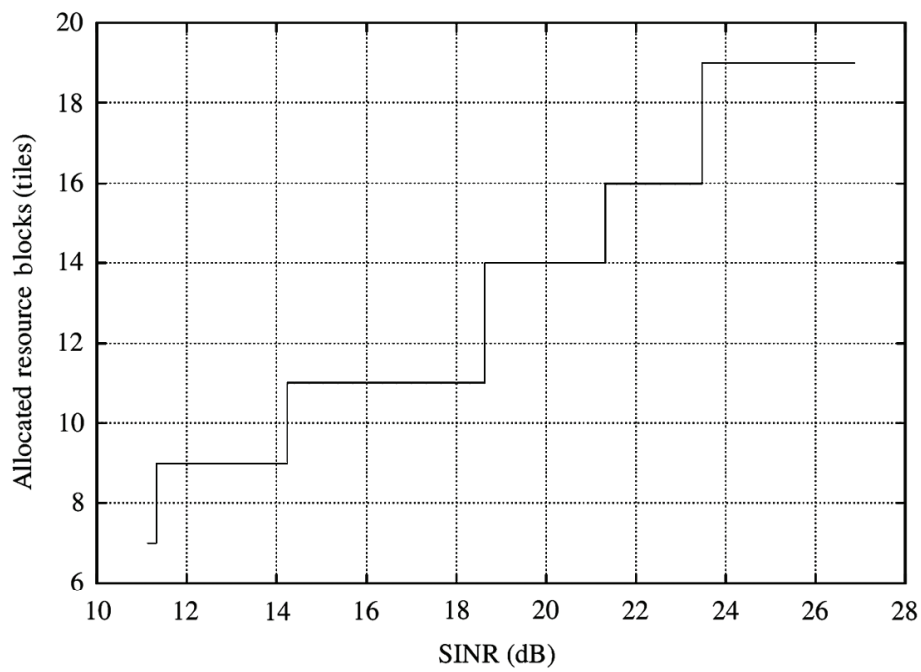

(c)

Figure 6. Performance metrics variations with SINR: (a) Maximum throughput rate per SINR; (b) SSR versus SINR; and (c) Allocated RBs (tiles) versus SINR.

mobility at the edges and handovers of users leaving the building. M-FCRA scheme outperforms FCRA due to reduced path loss hence improved SINR. However, FCRA performs better on the edge FAPs than M-FCRA as users are static and therefore FAPs kept traffic.

The plot of maximum throughput with SINR is illustrated in Fig. 6(a). As the SINR improves, throughput rate increases. This is because of reduced path loss in M-FCRA due to mobility of users as opposed to FCRA. M-FCRA achieves SINR up to $40 \mathrm{~dB}$, while the best for FCRA is about $28 \mathrm{~dB}$.

In Fig. 6(b), we plotted the SSR of M-FCRA and FCRA schemes as a function of SINR. We made two main observations. First, FCRA offers a constant SSR value. At convergence the SINR for FCRA scheme is constant because the users are static.

The network services on a constant SSR at each and every value of SINR as sent to FAPs by users. Second, SSR metric decreases with the increase of SINR for M-FCRA as there is an increase of interference level of each femtocell. According to the M-FCRA approach, fewer but densely populated clusters are created. Subsequently, the possibility of reutilization of the same RB (tile) among the formed clusters is decreased. M-FCRA does not allow reutilization of the same $\mathrm{RB} /$ tile within the same cluster.

The degree of interference in the network is inversely proportional to the number of allocated tiles. This is illustrated in Fig. 6(c). As SINR increases, the number of tiles allocated to each FAP increases exponentially. This shows that mobile users enjoy better QoS on an average as opposed to static users at poor or low SINR. Static users cannot improve SINR as they cannot move closer to serving FAP.

\section{Conclusion}

It is notable from this piece of work that user mobility has an impact in femtocell deployment; the higher the number of cells within the same location the more handover operations are executed. This problem can become critical because femtocells always require hard handover, which is, in general, a resource-demanding procedure. In this case, more resources will be required for numerous hardhandover operations, thus affecting the QoS to the end users. Again, a predictive approach for resource allocation to counteract this problem of resource allocation is of 
importance, and user mobility would be highly beneficial for the deployment of femtocells as presented in this work. Studies within this context remain of wide interest with explicit reference to femtocells and resource allocation and user mobility dynamics.

\section{References}

[1] J.G Andrews and V. Chandrasekhar, Femtocell networks: A survey, IEEE Communications Magazine, 46, 2008, 59-67.

[2] A. Hatoum, A. Nadjib, L. Rani, B. Raouf, and P. Guy, FCRA: Femtocell cluster-based resource allocation scheme for OFDMA networks, in IEEE Intern. Conf. on Communication, Kyoto, Japan, 2011.

[3] D. Lopez-Perez, A. Valcarce, G. de la Roche, and J. Zhang, Dynamic frequency planning versus frequency reuse schemes in OFDMA networks, in IEEE Veh. Technol. Conf., Barcelona, April 2009.

[4] M. Rahman and H. Yanikomeroglu, Enhancing cell-edge performance: A downlink dynamic interference avoidance scheme with inter-cell coordination, IEEE Transactions on WirelessCommunication, 9(4), 2010, 1414-1425.

[5] A. Attar, V. Krishnamurthy, and O.N. Gharehshiran, Interference management using cognitive base-station for UMTS LTE, IEEE Communications Magazine, 49(8), 2011, 152-159.

[6] J.W. Huang and V. Krishnamurthy, Cognitive base stations in LTE/3GPP femtocells: A correlated equilibrium gametheoretic approach, IEEE Transactions on Communications, 59(12), 2011, 3485-3493.

[7] S.-Y. Lien, C.-C. Tseng, K.-C. Cheng, and C.-W. Su, Cognitive radio resource management for QoS guarantees in autonomous femtocell networks, IEEE Int. Conf. Commun, Kyoto, Japan, May 2010.

[8] L. Tan, Z. Feng, W. Li, Z. Jing, and T.A. Gullier, Graph coloring based spectrum allocation for femtocell downlink interference mitigation, IEEE Wireless Commun. Netwk. Conf., Cancun, Mexico, March 2011.

[9] S. Uygungelen, G. Auer, and Z. Bharucha, Graph-based dynamic frequency reuse in femtocell networks, IEEE Veh. Technol. Conf., Budapest, Hungary, May 2011.

[10] Y.-S. Liang, W.-H. Chung, G.-K. Ni, I.-Y. Chen, H. Zhang, and S.-Y. Kuo, Resource allocation with interference avoidance in OFDMA femtocell networks, IEEE Transactions on Vehicular Technology, 61(5), 2012, 2243-2255.

[11] K. Sundaresan and S. Rangarajan, Efficient resource management in OFDMA femtocells, Proc. ACM MobiHoc, New York, USA, May 2009

[12] H.-C. Lee, D.-C. Oh, and Y.-H. Lee, Mitigation of interfemtocell interference with adaptive fractional frequency reuse, IEEE Int. Conf. Commun., Cape Town, South Africa, May 2010.

[13] V. Chandrasekhar and J. Andrews, Spectrum allocation in tiered cellular networks, IEEE Transactions on Communications, 57(10), 2009, 3059-3068.

[14] L.G.U. Garcia, K.I. Pedersen, and P.E Mogensen, Autonomous component carrier selection: Interference management in local area environment for LTE-advanced, IEEE Communiations Magazine, 47(9), September 2009, 110-116.

[15] R. Langar, R. Bontaba, and N. Bouabdallah, Design and analysis of mobility-aware clustering algorithms for wireless mesh networks, IEEE/ACM Transactions on Networking, 18(6), 2010, 1677-1690.

[16] D. Lopez-Perez, A. Valcare, G. da la Roche, and J. Zhang, OFDMA femtocells: A roadmap on interefernce avoidance, IEEE Communiations Magazine, 47(9), September 2009, $41-48$.

[17] F. Capozzi, G. Piro, L.A. Grieco, G. Boggia, and P. Camarda, On accurate simulations of LTE femtocells using an open source simulator, EURASIP Journal on Wireless Communication and Networking, vol. 2012:328, 2012, 1-13.

[18] E. Dahlman, S. Parkvall, J. Skold, and P. Beming, $3 G$ evolution HSPA and LTE for mobile broadband (USA: Academic Press, 2008).
[19] 3GPP, Technical Spec. Group Radio Access Network - Physical Channel and Modulation (Release 8) 3GPP TS 36.211, 3GPP, 2008.

[20] D. Winner II, Winner II channel models part I - channel models, Technical Report, September 2007.

[21] S. Bellahsene and L. Kloul, A new Markov-based mobility prediction algorithm for mobile networks, Bertinoro, Italy, 2010.

[22] S. Bellahsene, L. Kloul, and D. Barth, A hierarchical prediction model for two nodes-based ip mobile networks, 12th MSWiM, Canary Islands, Spain, 2009.

[23] B. Dominique, C. Amira, L. Kloul, and M. Oliver, Femtocells sharing management using mobility prediction model, 16th ACM Intern. Conf. on Modeling, Analysis and Simulation of Wireless and Mobile Systems, ACM, New York, NY, USA, July 2013.

[24] 3GPP, Simulation assumptions and parameters for FDD HenB RF requirements, 3GPP TSG RAN TS 36.331, 3GPP, 2009.

[25] 4G++, Advanced Performance Boosting Techniques in 4th Generation Wireless System (online).

[26] IBM, http://www.ilog.com/products/cplex/

\section{Biographies}

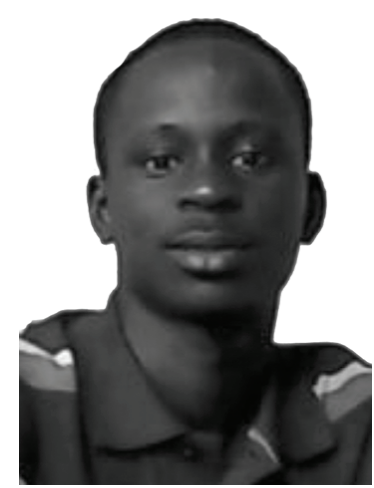

Nicholas O. Oyie is currently a graduate assistant at Masinde Muliro University of Science and Technology (MMUST) in the Department of Electrical and Communication Engineering. He received his B.Tech. in electrical and communication engineering from the same institution in 2009; and currently working toward M.Sc. in telecommunication engineering at Jomo Kenyatta University of Agriculture and Technology, Kenya. In 2009-2010, he was a BSS engineer with Essar Telecom Kenya working on project management, systems and transmission links. His research areas of interest include radio resource and mobility management in wireless networks, femtocells cells and quality of service support.

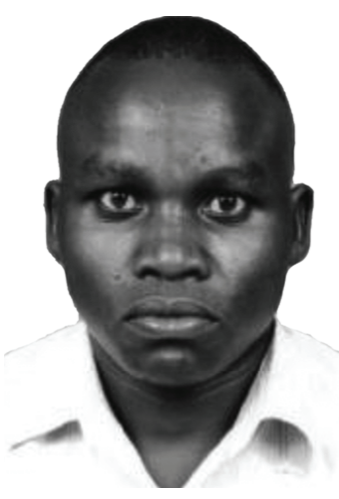

Philip K. Langat is currently the Chairman, Department of Telecommunication and Information Engineering at the Jomo Kenyatta University of Agriculture and Technology, Kenya. He received his Ph.D. and M.Sc degrees from Stellenbosch University, South Africa and Telecommunication Engineering, JKUAT, Kenya, respectively. $\mathrm{He}$ is a member of Engineers Registration Board of Kenya (EBK) and Kenya Society of Electrical and Electronic Engineers (KSEEE). He has research interest in electromagnetics: computational electromagnetic EM modelling techniques (FEM, FDTD and MoM), applied antenna theory: microstrip antennas and microstrip antenna array design, electromagnetic compatibility (EMC) and radio frequency interference (RFI) mitigation strategies, high frequency metrology, computer skills for 
numerical simulation and programming: MATLAB, Microwave Office, CST Studio and FEKO, applied electromagnetics, radiation and scattering problems, $\mathrm{RF}$ and EM computations, EMI/EMC, RFI mitigation, and multiantenna communications and applications.

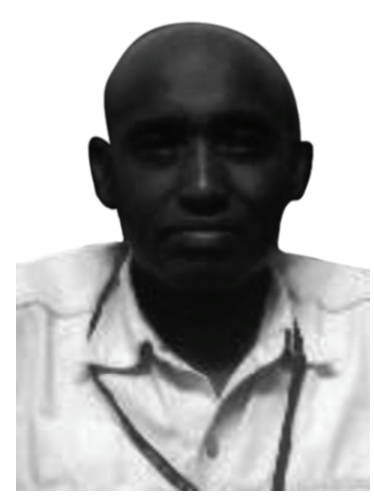

Stephen Musyoki received his Ph.D. and M.Sc. degrees from University of ElectroCommunications, Japan in 1985 and University of Sierra Leone in 1977, respectively. He is currently a Professor and Director in the Department of Electrical and Electronic Engineering at Technical University of Kenya. He has been the chairman of the Department of Telecommunication and Information Engineering at Jomo Kenyatta University of Agriculture and Technology. He has worked at Sangikyo Corporation, Japan (April 2000-October 2002) as senior department engineer, NEC Corporation, Japan (April 1994-March 2000) as a software research and development engineer, Japan Atomic Energy Research Institute, Japan (April 1992-March 1994) as a research fellow, Teachers Service Commission, Kenya (December 1981-March 1991) as a senior lecturer, Electronics and Telecommunications and Ministry of Information and Broadcasting, Kenya (October 1977-November 1981) as a lecturer, Radio and Television Broadcasting Technology. His research interests include signal communication and digital communications. He is a member of Kenya Engineers Registration Board (EBK) and Institution of Electrical and Electronics Engineers (New York, USA). 